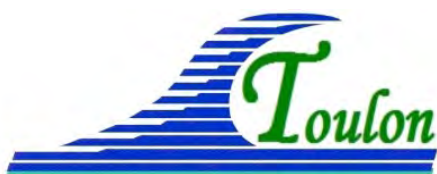

\title{
Analyse des données AIS des secteurs PACA Est et Sanctuaire PELAGOS pour l'évaluation des problématiques liées au trafic maritime pour la gestion du milieu marin
}

\author{
François CARNUS ${ }^{1}$, Ann Sophie GABELLINI ${ }^{2}$, Olivier MERCIER ${ }^{3}$
}

1. BRL Ingénierie, Directeur de projets en environnement littoral et marin, 1105 avenue Pierre Mendès France 30001 Nîmes Cedex 5, France. francois.carnus@brl.fr

2. BRL Ingénierie, Ingénieur d'études en aménagement littoral, 1105 avenue Pierre Mendès France 30001 Nîmes Cedex 5, France. ann-sophie.gabellini@brl.fr

3. BRL Ingénierie, Ingénieur en informatique et SIG, 1105 avenue Pierre Mendès France 30001 Nîmes Cedex 5, France. olivier.mercier@brl.fr

\section{Résumé :}

Le secteur Est de la région PACA ainsi que le Sanctuaire PELAGOS constituent des écrins de la biodiversité marine méditerranéenne qu'il est primordial de préserver compte tenu des fortes pressions anthropiques qui impactent ces milieux tels que les différents trafics maritimes. Afin de mieux appréhender ces problématiques, l'Agence Nationale des Aires Marines Protégées (AAMP) a souhaité dresser l'état des lieux des activités et des trafics maritimes sur ces deux secteurs à travers l'analyse des données AIS (Automatic Identification System) d'identification et de localisation des navires. L'étude menée par BRLi s'est focalisée sur les deux besoins suivants : (i) PACA Est : analyse géographique, quantitative et qualitative de la fréquentation des côtes par les navires de plaisance avec un focus sur la grande plaisance (navires $>24 \mathrm{~m}$ ), afin d'aider à la définition de mesures de gestion de plusieurs aires marines protégées. (ii) Sanctuaire PELAGOS : cartographie de la distribution des navires et de leurs caractéristiques (vitesse, taille, type), afin d'aider à l'évaluation de l'impact des activités humaines sur les mammifères marins.

Mots-clés: Données AIS, Trafic maritime, Aires marines protégées, Environnement marin.

\section{Contexte de l'étude}

\subsection{Rappel des objectifs de l'étude}

La mission de BRLi a consisté en l'analyse des données AIS fournies par le CEREMA sur les années 2012 et 2013, de façon à évaluer et analyser les éléments suivants : 


\section{Thème 6 - Gestion durable des zones littorales et estuariennes}

- Sur le secteur PACA Est: identification des routes principales des navires de plaisance et de croisière, identification des zones de mouillage privilégiées des navires de plaisance et de croisière, zooms sur les pratiques de mouillage des navires de plaisance au sein des différentes Aires Marines Protégées.

- Sur le secteur du sanctuaire PELAGOS : identification des routes principales de tous les types de navires et croisement avec les données disponibles sur les présences de mammifères marins.

Cet article et les extraits de l'atlas cartographique associés présentent la méthodologie mise en œuvre, une analyse de la représentativité des données AIS et les principaux résultats obtenus.

\subsection{Focus sur le système AIS}

Le système d'identification automatique "AIS" est un système d'identification et de suivi autonome et continu des navires, permettant d'échanger par voie électronique avec les autres navires à proximité et avec les autorités à terre les données du navire, et notamment son identification, sa position, son cap et sa vitesse. A noter qu'il n'existe pas a priori de texte fixant réglementairement à partir de quelle longueur minimale les navires doivent être munis d'un système AIS. Pour cette étude, il a été pris l'hypothèse d'une longueur minimale de $15 \mathrm{~m}$ : les navires d'une longueur inférieure sont considérés comme la "petite plaisance" ne possédant pas de balise AIS, et ne sont donc pas pris en compte dans les analyses du trafic et des mouillages.

\section{Méthodologie et analyse des données AIS}

La première étape a consisté en l'analyse des données AIS mises à disposition par le CEREMA afin d'en évaluer la répartition et la continuité dans le temps et dans l'espace. Les étapes suivantes, d'intégration et de traitement, ont permis d'affiner cette évaluation.

\subsection{Etape 1 : analyse des données sources}

\section{Représentativité temporelle}

Les données sources analysées concernent les années 2012 et 2013 sur une période cumulée de 20 mois (4 mois manquants). Ces données ont été fournies sous la forme de nombreux fichiers textes, chaque fichier ne concernant qu'une journée. Leur représentativité temporelle a été analysée. Les conclusions sont les suivantes :

- Sur une période de deux ans (2012/2013), 12\% des fichiers-jours sont absents.

- En 2013, 24\% des fichiers journaliers de la haute saison touristique sont indisponibles.

- Une suspicion concernant l'exhaustivité des enregistrements des données et positions des navires persiste pour au moins 5\% des fichiers (sur les deux années). 


\section{XIV èmes Journées Nationales Génie Côtier - Génie Civil \\ Toulon, 29 juin au $1^{\text {er }}$ juillet 2016}

\section{Représentativité spatiale}

La zone géographique analysée couvre totalement la zone de PACA Est et la partie française du Sanctuaire PELAGOS mais n'englobe pas sa totalité.

Nature des données

Chaque ligne de données rassemble les informations principales suivantes :

- "Temps" : en jours/mois/années heures : minutes : secondes,

- "IdNav" : identifiant (crypté) du navire,

- "Long_X" : longitude en degrés, avec 4 décimales (précision : 10 m environ),

- "Lat_Y" : latitude en degrés, avec 4 décimales (précision : 10 m environ),

- "VistFond" : vitesse de fond en nœuds,

- "Route" : direction en degrés (cap du navire),

- "TypeNav" : catégorie de navire,

- "Destinat" : destination, peut varier au cours d'un trajet

- "Longueur" : longueur en mètres du navire (nb : pas constant !),

- "Tirant" : tirant d'eau en mètres.

Pour cette étude, ce sont exclusivement les champs "Temps", "IdNav", "Long_X", "Lat_Y", "Longueur" et "TypeNav" qui ont été analysés.

\subsection{Etape 2 : traitement d'intégration}

Le nombre initial d'enregistrements AIS analysés est d'environ 52 millions. Afin d'optimiser et de faciliter les traitements et les représentations cartographiques, l'étape d'intégration a été précédée d'un filtrage et d'un nettoyage des données. A l'issue de ces traitements, les fichiers de données se sont révélés encombrés de positions statiques. L'élimination de ces enregistrements permet une économie volumique importante, de l'ordre de 50\%. D'autre part, les traitements permettent de détecter des anomalies d'enregistrement et de codage de l'information (date/heure, positions improbables) ainsi que des lacunes dans les données (exemple : champs "TypeNav" et "Longueur" non renseignés).

\subsection{Etape 3 : typologie et analyse quantitative}

L'analyse du champ relatif au type de navire permet de dresser la typologie des navires présents dans la zone et consiste à compter le nombre de positions pour chaque type. Au total pour l'année 2012, le nombre d'enregistrements inutilisables, qui était de 8.6\% avant les corrections, est ramené à 6.4\% (407 919 enregistrements). Pour l'année 2013, ce nouveau taux est de 5.2\% (386 998 enregistrements). Ceci pose, encore une fois, le problème de la fiabilité des données AIS enregistrées dont les causes peuvent être des erreurs de transmissions, erreurs d'identification dans le navire, erreurs dans l'identification du navire... Les traitements réalisés permettent de corriger certaines de ces erreurs. On peut donc noter que : 
- Les deux catégories les plus représentées et donc naviguant le plus sont les cargos (33.1\%) et les navires à passagers (31.5\%), dont les longueurs sont majoritairement supérieures à $80 \mathrm{~m}$,

- Suivent les tankers (10.7\%) et les navires de plaisance (12.2\%), dont les longueurs sont respectivement supérieures à $80 \mathrm{~m}$ et comprises entre 24 et $50 \mathrm{~m}$,

- Toutes les autres catégories restent minoritaires (entre 0 et $2 \%$ ), à noter néanmoins que la catégorie "autre" rassemblant les types "inconnus" reste non négligeable (5.6\%).

\subsection{Etape 4 : localisation des données}

En analysant, pour chaque navire en mouvement (vitesse $>6$ nœuds), le temps écoulé entre deux enregistrements successifs, il est possible de localiser les zones où les transmissions sont moindres (Cf. figure 1 pour l'année 2012). Il y a en fait trois zones pour lesquelles les fréquences des réceptions sont réduites : à l’Ouest de la Corse entre la Sardaigne et Marseille, tout l'Est de la Corse, et au niveau de la façade italienne, entre l’Ile de Gorgona et la côte.

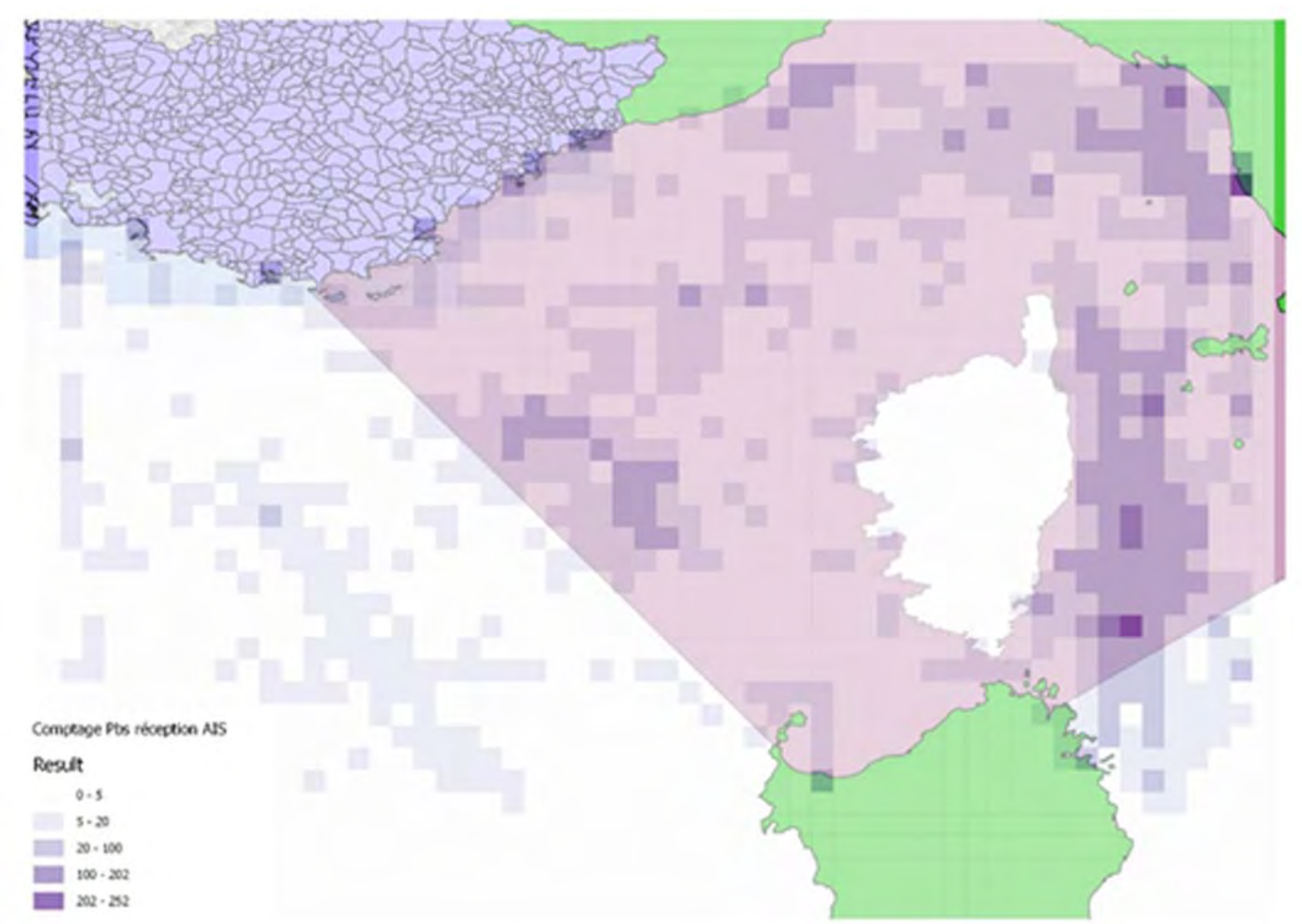

Figure 1. Trous géographiques de réception en 2012. 


\section{XIV ${ }^{\text {èmes }}$ Journées Nationales Génie Côtier - Génie Civil \\ Toulon, 29 juin au $1^{\text {er }}$ juillet 2016}

\subsection{Synthèse quant à la représentativité des données AIS}

Comme décrit ci-avant, différents traitements/corrections ont été nécessaires pour rendre "utiles et utilisables" les données AIS. Pour les années 2012 et 2013, le nombre d'enregistrements utiles et utilisables, après corrections, est respectivement de 5971801 et de 12589 072. L’"efficience" des données par rapport aux fichiers sources, auxquels sont soustraits les points hors-zone, est donc de 31.7\% pour 2012 et $42.8 \%$ pour 2013. De nombreuses erreurs perturbent par ailleurs l'exploitation des données. En conclusion, même s'il avère que la base de données AIS est une source de données très appréciable, la fiabilité du système semble être fragilisée par plusieurs facteurs :

- le nombre de jours de carence en données est important : environ 10\% du temps sur les années 2012 et 2013,

- les nombreuses altérations dans la qualité des données transmises/enregistrées concernant environ $10 \%$ des données sont à déplorer. Les détections et les identifications de ces anomalies sont coûteuses en temps de traitement et d'analyse. Les corrections ne sont possibles que dans un quart (environ) des cas. Elles sont également très longues à réaliser,

- la qualité de la transmission/réception n’est pas homogène sur la zone : il existe des zones où la transmission est moins fiable.

D'autre part, il est important de noter que l'absence de balise AIS sur les petits bateaux (bateaux de plaisance et voiliers de 5 à $15 \mathrm{~m}$ par exemple) ne permet pas d'apprécier la totalité du trafic maritime, ni de quantifier de manière précise la fréquentation des sites protégés et des aires de mouillage.

\section{Analyse du trafic maritime sur le secteur PACA Est}

Nous focalisons uniquement sur les navires de plaisance (types AIS = navires de plaisance + voiliers) et les navires de croisières (types AIS = navires à passagers + navires à grande vitesse). L'étendue géographique concernée correspond à la partie littorale PACA Est depuis la Rade de Marseille à la frontière italienne.

\subsection{Analyse des routes principales}

Les éléments suivants peuvent être retenus :

- la navigation de plaisance se concentre le long de la côte, elle est essentiellement représentée (via les données AIS) par des navires de longueur comprise entre 24 et $50 \mathrm{~m}$,

- la navigation de croisière est à la fois côtière et traversante (Corse, Espagne, Maghreb), elle est essentiellement représentée par des navires de longueur supérieure à $80 \mathrm{~m}$,

- les taux de trafic plaisance/croisière sont du même ordre de grandeur avec une légère prédominance de la croisière (respectivement $44 \%$ et $56 \%$ ), malgré un nombre de navires 3 fois supérieur pour la plaisance, 


\section{Thème 6 - Gestion durable des zones littorales et estuariennes}

- le trafic de plaisance est multiplié par 10 de la basse à la haute saison, tandis que le trafic de croisière est multiplié par 3.

\subsection{Analyse des zones de mouillage}

L'identification des zones préférentielles de mouillage en PACA Est résulte d'une suite de traitements, dont les étapes sont les suivantes :

- identification du trait de côte et extraction des positions en mer (hors ports),

- pour chaque itinéraire, identification des positions successives où la vitesse est inférieure à 1.7 nœuds puis incrémentation des séquences,

- identification des temps de début et de fin de séquence (calcul de la durée de mouillage, et calcul des positions moyennes et des écarts-types),

- filtrage sur les séquences dont la durée est supérieure ou égale à 20 minutes (0.3h) et dont l'écart-type est inférieur à $0.005^{\circ}$.

A l'issu de ces traitements le nombre de mouillage dénombré apparait faible sur l'ensemble de la haute saison (de mai à septembre 2013) : environ 6000 mouillages sur toute la côte PACA Est pour les 5 mois de la haute saison, soit en moyenne 40 mouillages par jour valeur qui semble largement sous-estimée. Il est donc pertinent de s'interroger quant à la fiabilité des données AIS pour quantifier l'activité de mouillage puisque :

- seuls les "grands" navires possèdent le système : la petite plaisance (longueur < 15m) reste écartée de l'analyse bien qu'elle représente une large part des activités de cabotage estival et de mouillage,

- il existe de nombreuses erreurs de transmission des données,

- certains navires éteignent leur système une fois au mouillage ce qui rend impossible l'analyse.

Néanmoins, les données AIS permettent une pré-analyse qualitative de l'activité de mouillage en renseignant quant aux zones géographiques et aux périodes les plus fréquentées.

\section{Analyse du trafic maritime sur le secteur du Sanctuaire PELAGOS}

L'analyse concerne tous les types de navires représentés par les données AIS (plaisance, croisière, commerce, activités maritimes, activités portuaires et activités étatiques). L'étendue géographique correspond à l'emprise du Sanctuaire PELAGOS. Dans un premier temps, il a été analysé la durée cumulée de présence des navires dans le sanctuaire sur la période entière (2012-2013). Les différentes routes prépondérantes sont ainsi mises en évidence : navigation côtière, Marseille/Toulon - Bastia, Marseille/Toulon - Ajaccio, Marseille/Toulon - Bonifacio ou Sardaigne, Gênes Corse/Sardaigne/PACA, La Spézia - Corse/Sardaigne/PACA. Dans un second temps, il a été analysé les routes principales des navires (tout type confondu) selon leur 


\section{XIV ${ }^{\text {èmes }}$ Journées Nationales Génie Côtier - Génie Civil Toulon, 29 juin au $1^{\text {er }}$ juillet 2016}

dangerosité vis-à-vis des mammifères marins. Les classes de dangerosité des navires ont été établies en fonction de leurs longueurs et vitesse, selon la matrice de la figure 2.

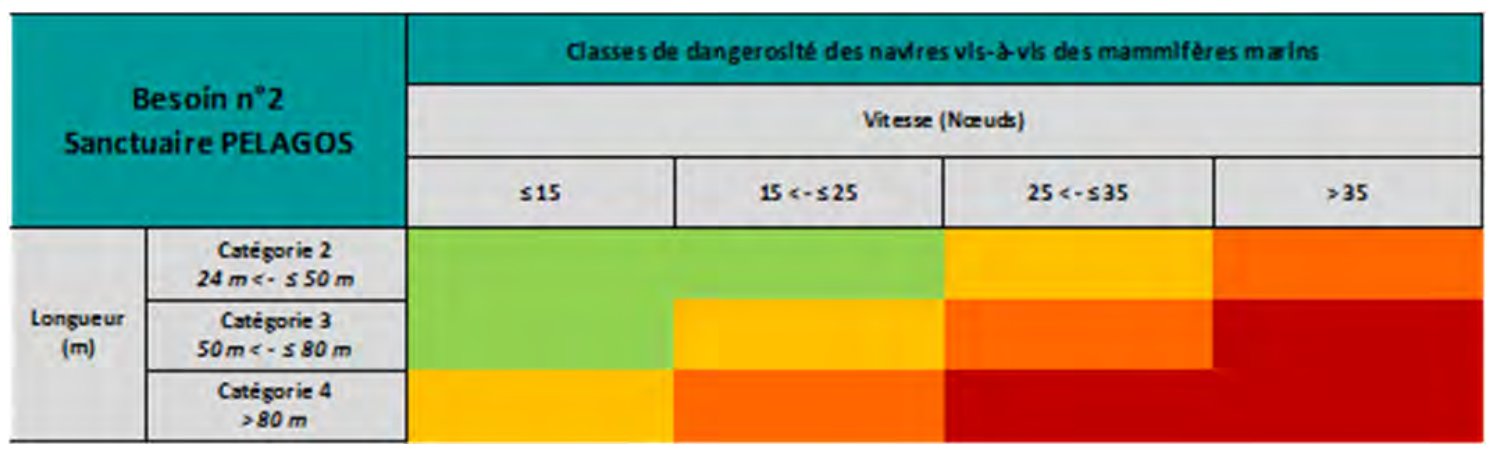

Figure 2. Classes de dangerosité des navires vis-à-vis des mammifères marins.

\section{Atlas cartographique}

Des extraits de l'atlas cartographique illustrant les résultats de cette étude sont présentés ci-après (Cf. figures 3 et 4).

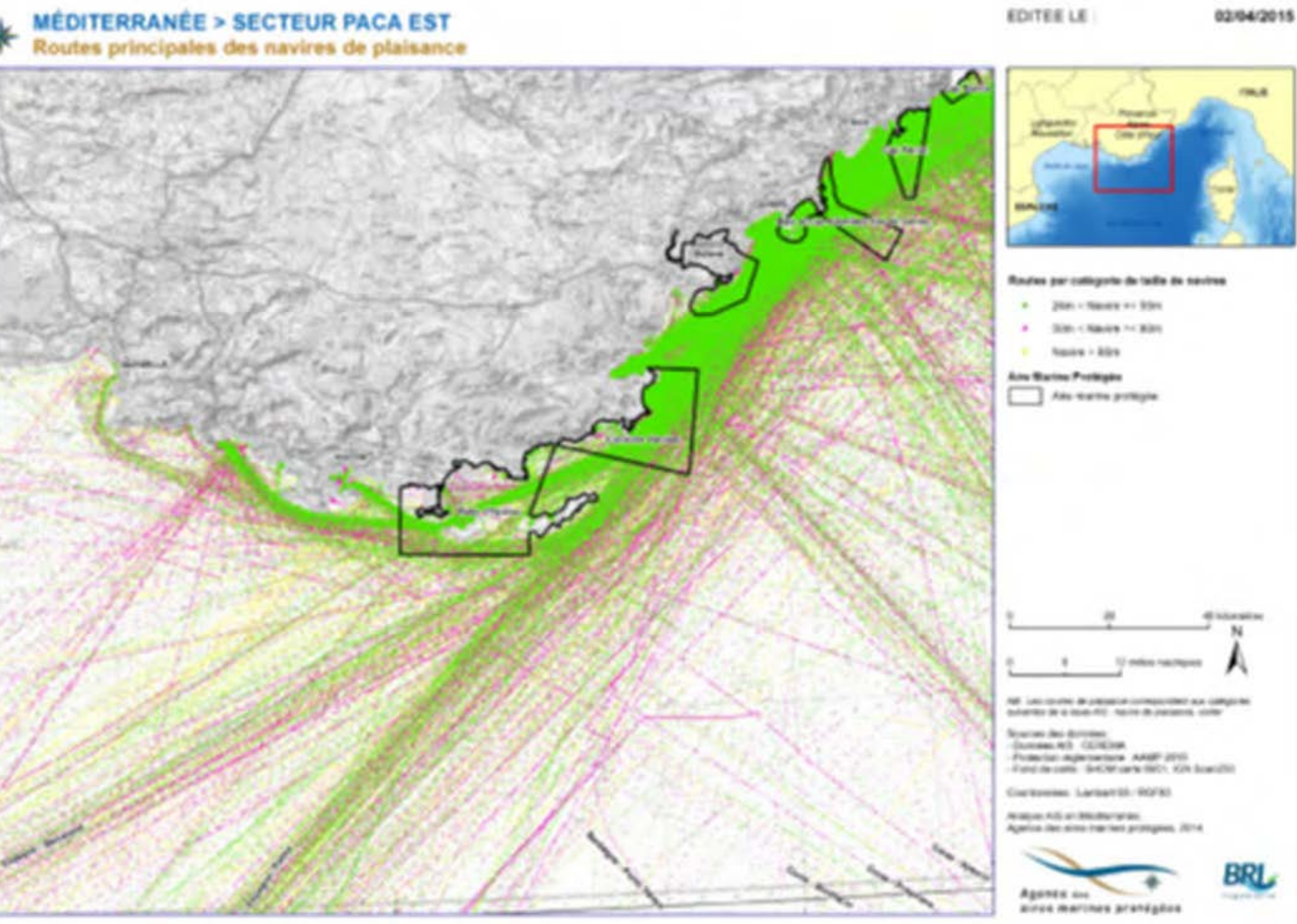

Figure 3. Routes principales des navires de plaisance en PACA Est. 


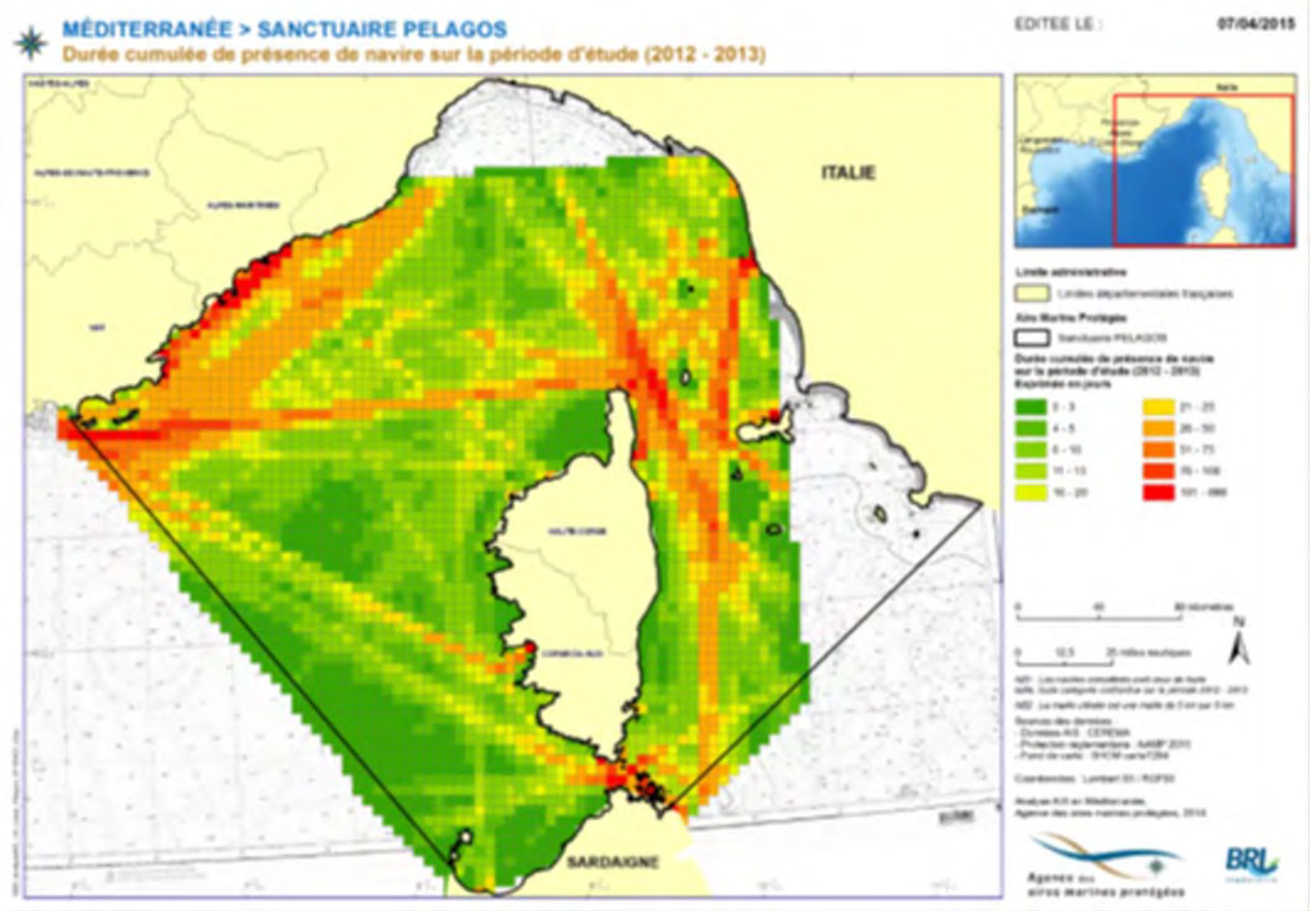

Figure 4. Durée cumulée de présence de navires sur la période d'étude au sein du Sanctuaire PELAGOS.

\section{Conclusion}

En conclusion, même s’il avère que la base de données AIS est une source de données très appréciable, la fiabilité du système semble être fragilisée par plusieurs facteurs :

- Le nombre de jours de carence en données est important : environ 10\% du temps sur les années 2012 et 2013,

- De nombreuses altérations dans la qualité des données transmises/enregistrées concernant environ $10 \%$ des données sont à déplorer,

- La qualité de la transmission/réception n’est pas homogène sur la zone : il existe des zones où la transmission est moins fiable.

D'autre part, il est important de noter que l'absence de balise AIS sur les petits bateaux (bateaux de plaisance et voiliers de 5 à $15 \mathrm{~m}$ par exemple) ne permet pas d'apprécier la totalité du trafic maritime, ni de quantifier de manière précise la fréquentation des sites protégés et des aires de mouillage.

Néanmoins, les données AIS permettent une pré-analyse qualitative de l'activité de mouillage et des routes maritimes empruntées en renseignant quant aux zones géographiques et aux périodes les plus fréquentées. 